\title{
Editorial
}

\section{Day Care Surgery: An Overview}

Outpatient surgery and anesthesia, which started as a money-saving modality, has picked up momentum even in India over the last two decades due to innovations in surgical techniques and advances in anesthesia. The present generation is one for whom time is as precious as money, and therefore, early discharge is popular. Positive feedback from patients has increased the popularity of outpatient surgery. Based on a number of studies and the current practice in India and in Western countries, a number of operative procedures on selected patients from all age groups can be conducted on a day care basis either in standalone day surgical units (DSUs) or ambulatory care centers (ASCs) or in a hospital setting. However, patient safety should never be compromised in the name of "fast track-

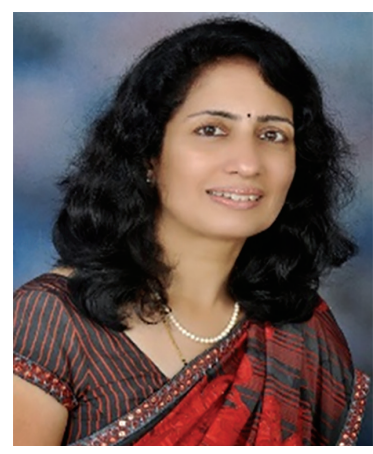
ing" and cost containment. For hospitals, a shift to day surgery is effectively a cultural revolution. The development of day surgery is an indication of how extramural hospitals and clinics will develop in future; inpatient surgical beds will gradually need to be converted to day care beds. ${ }^{1}$

Day surgery will improve facilities' performance levels and will allow for organizational and financial room for maneuver, while ensuring that patients are at the center of the new system.

The history dates back to the early 20th century when James Nicoll, a Glasgow surgeon, performed almost 9,000 outpatient operations on children in 1903. In 1912, Ralph Waters from Iowa, the United States, described "The Down Town Anaesthesia Clinic," where he gave anesthesia for minor outpatient surgery. However, over the next two decades the concept lost its momentum. The first hospital-based ambulatory unit developed in 1960 in the United States. ${ }^{2}$

"A surgical day case is a patient who is admitted for an operation on a planned nonresident basis and who nonetheless requires facilities for recovery. The whole procedure should not require an overnight stay in a hospital bed." ${ }^{3}$

There are some terms used synonymously among day care surgeons. Day care surgery is when a patient recovers from surgery and is fit to return home within a day. Ambulatory surgery is when the patient returns home the same day in the evening, and the term "office surgery" is used for a patient fit to return home within a few hours (Table 1). ${ }^{1}$

Day surgery improves quality of care by creating a situation in which everything is arranged around the patients and their interests: Paramedics, nurses, surgeons, anesthetists, and administrative staff have to work together to create a care pathway that involves no wasted time and has no discontinuity. Continuity of care remains essential, just as it is for inpatient admissions. ${ }^{4}$ Physicians, anesthetists, and surgeons have made and continue to make progress in risk management. They have reduced recovery time and have restored patients' autonomy sooner, by providing better prevention and treatment of pain, bleeding, nausea, and urinary problems, which previously used to keep patients in the hospital for several days.

The International Association for Ambulatory Surgery (IAAS) was founded in 1995 with the aim to promote the development and growth of high-quality ambulatory surgery worldwide. Formal development of ambulatory anesthesia occurred with the establishment of the "Society for Ambulatory Anesthesia" (SAMBA) in 1984 and the evolution of the postgraduate subspecialty training program.

Ambulatory surgery can be practised at various levels. It could be minor ambulatory surgery (under local anesthesia), major ambulatory surgery (under GA, central neuroaxial block with or without IV sedation), or in-patient ambulatory surgery. ${ }^{5}$

Table 1: Definitions of terms in day surgery ${ }^{3}$

\begin{tabular}{ll}
\hline English terminology & Synonym/definition \\
\hline Ambulatory & Day, same day, day only \\
Extended recovery & 23 hours, overnight stay, single night \\
Short stay & - \\
Ambulatory surgery center (facility) & $\begin{array}{l}\text { A center (facility) designed for the optimum management of an ambulatory surgery/procedure } \\
\text { patient }\end{array}$ \\
Ambulatory surgery/procedure & $\begin{array}{l}\text { An operation/procedure, excluding an office/surgery or outpatient operation/procedure, where } \\
\text { the patient is discharged on the same working day }\end{array}$ \\
\hline
\end{tabular}

(Source: International Association for Ambulatory Surgery, Ambulatory (day) surgery. Suggested international terminology and definitions. London: IAAS; 2003) 
Day surgery is most useful for routine and common surgical procedures. There are medical and surgical selection criteria. It must be possible to carry out the procedure safely. A case-by-case assessment should be done considering the particular combination of patient, procedure, and organization. The length of surgery, the postoperative monitoring period, and the time for early rehabilitation are important factors. Other considerations are the length of functioning of the center, predictions made on postoperative progress, and the ASA American Society of Anesthesiologists status. ${ }^{6}$

What about the procedure? Surgical procedures suitable for ambulatory surgery should be accompanied by minimal postoperative physiologic disturbances and an uncomplicated recovery. The primary predictors of prolonged stay or unanticipated admission after day-case surgery are related (Table 2) to the surgical procedure [e.g., blood loss, pain, postoperative nausea and vomiting (PONV)]. An alternative to same-day surgical concept is a planned overnight admission to the hospital after surgery. This approach (AM admit, 23 hours, short stay, come and stay) is often classified as outpatient surgery and has preserved many of its advantages. Table 2 enumerates the common procedures done as day surgery. ${ }^{7}$

The duration of surgery in the ambulatory setting was originally limited to procedures lasting less than 90 minutes because investigators have found that the operating and anesthetic time is a strong predictor of postoperative complications (e.g., pain, emesis) and delayed discharge, as well as unanticipated admission to the hospital after ambulatory surgery.

Some important exclusion criteria exist for day surgery. While most of them are undebatable, there are a few that can be considered on an individual case assessment (Table 3). ${ }^{7}$

The advantages are innumerable. Patient preference, especially for children and the elderly, lack of dependence on the availability of hospital beds, greater flexibility in scheduling operations with low morbidity and mortality, and

Table 2: Common procedures done as day surgery

\begin{tabular}{ll}
\hline Specialty & Types of procedures \\
\hline Dental & Extraction, restoration, facial fractures \\
Dermatology & Excision of skin lesions \\
General & $\begin{array}{l}\text { Biopsy, endoscopy, excision of masses, hemorrhoidectomy, herniorrhaphy, laparoscopic procedures, } \\
\text { varicose vein surgery } \\
\text { Cone biopsy, dilatation and curettage, hysteroscopy, laparoscopy, polypectomy, tubal ligation, } \\
\text { vynecology }\end{array}$ \\
vaginal hysterectomy \\
Cataract extraction, chalazion excision, nasolacrimal duct probing, strabismus repair, tonometry \\
Orthopedic & $\begin{array}{l}\text { Anterior cruciate repair, arthroscopy, bunionectomy, carpal tunnel release, closed reduction, } \\
\text { hardware removal, manipulation under anesthesia }\end{array}$ \\
Otolaryngology & Adenoidectomy, laryngoscopy, mastoidectomy, myringotomy, polypectomy, rhinoplasty, tonsillectomy, \\
tympanoplasty & Chemical sympathectomy, epidural injection, nerve blocks \\
Plastic surgery & Basal cell cancer excision, cleft lip repair, liposuction, mammaplasty, otoplasty, scar revision, \\
Urology & septorhinoplasty, skin graft \\
& Bladder surgery, circumcision, cystoscopy, lithotripsy, orchiectomy, prostate biopsy, vasovasostomy \\
\hline
\end{tabular}

Table 3: Exclusion criteria for outpatient surgery

\section{l Medical}

a Unfit ASA IV, ASA III

b Obese: Body mass index $>35$

c Nature of pathology: Large scrotal hernias major intrathoracic intraabdominal or intracranial surgery

d Procedures requiring more than one hour

e Surgery expected to have major fluid or blood loss

II Patient

a Concept of day care surgery unacceptable to the patient

b Psychologically unstable

c If patient lives far away from the hospital

d Infants $<3$ months of age and preterm babies

III Social: No competent relative or friend to

i) Accompany or drive patient home after operation

ii) Look after him or her at home for the next 24-48 hours 
Table 4: The following factors are contributory to PONV ${ }^{1}$

\begin{tabular}{ll}
\hline Patient factors: & $\begin{array}{l}\text { Young, obese females at the preovulatory stage of menstrual cycle, history of PONV/motion sickness, } \\
\text { pregnancy. }\end{array}$ \\
Surgical factors: & Eye, ENT, suction D\&C, Laparoscopy, orchidopexy \\
Anesthetic/Analgesics: & Opiates, nitrous oxide, volatile Inhalational agents \\
Anesthesiologist: & Good or bad \\
\hline
\end{tabular}

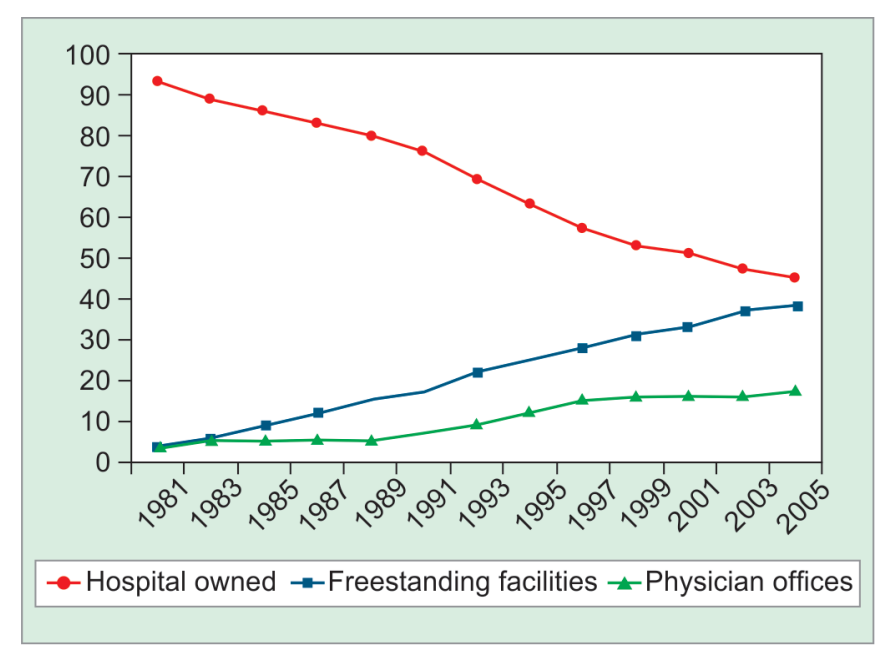

Graph 1: Percent of outpatient surgeries by facility type, 1981-2005

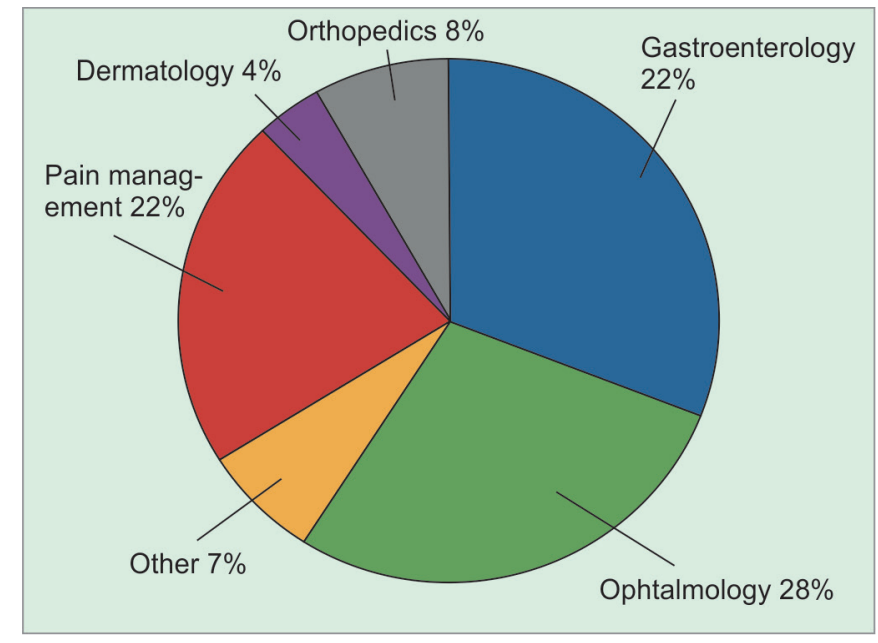

Graph 2: Specialty-wise cases done in 2010 (US) ${ }^{9}$

lower incidence of infection and respiratory complications are known with day surgery. A much higher volume of patients (greater efficiency) can be attended to while the surgical waiting lists are shortened. There is lower overall procedural costs with less preoperative testing and postoperative medication. ${ }^{8}$

The most important factors that lead to the success of same day discharge are patient selection, patient information, preoperative assessment, proper anesthetic and postanesthetic care, patient acceptability, and audit. Postoperative nausea and vomiting is the single most important deterrent that if controlled or prevented effectively can make the management of day surgery easier. The following factors are contributory to PONV (Table 4). ${ }^{1}$

The American Hospital Association published the rising trend of day surgeries performed across hospitals in the United States (Graphs 1 and 2) over 14 years. As is evident, the number of day surgeries done in freestanding facilities and outpatient offices were on a rising trend, while the number performed in hospitals showed a downward trend (Graphs 1 and 2 ). ${ }^{9-11}$

On the downside, postoperative complications do occur and there is fragmentation of care. The reduced observation time is also to be considered, and some patients require emergency readmission albeit at a low rate. It is prudent for ambulatory care facilities to be prepared for such complications and have an admission arrangement with a back-up facility for untoward occurrences.

To summarize, the continued growth in ambulatory surgery is related to expansion in minimally invasive surgical techniques and office-based procedures. Preexisting medical conditions are rarely an exclusion criterion for ambulatory surgery. The choice of anesthetic technique has a significant effect on postoperative side effects and discharge time.

Monitored anesthesia care is associated with the fewest side effects and the shortest time to discharge. We also need to fund progress and research (e.g., robot-guided or minimally invasive surgery, endovascular surgery, telemedicine, biotherapies, etc.) and to improve quality of care still further. It is therefore essential to create room for maneuver, and the development of day surgery will enable this. 


\section{REFERENCES}

1. Wig J. The current status of day care surgery: a review. Indian J Anesth 2005;49(6):459-466.

2. Waters RM. The down town anesthesia clinic. Am J Surg 1919;(Suppl 33):71-73.

3. Newson CD, Nathanson MH, White PF. Anaesthesia for day-stay (come and go) surgery. In: Healy TEJ, Cohen PJ, editors. Wylie and Churchill - Davidson's : a practice of anaesthesia. 6th ed. London: Edward Arnold; 1995. p. 1363-1390.

4. Bapat R, Kanthari CV, Rank S, Bakshi G, Iyer A. Day care surgery in a public hospital set up (personal communication); 2000.

5. Henderson AJ. Ambulatory surgery: Past, present, and future. In: Wetchler BV, editor. Anesthesia for ambulatory surgery. Philadelphia (PA): Lipincott; 1991. p. 1-27.

6. Federated Ambulatory Surgery Association. Special study. Alexandria (VA): Federated Ambulatory Surgery Association; 1986.

7. Ambulatory Surgery Centers. Encyclopedia of surgery [Internet]. Senagore AJ, editor. Thomson gale; 2004.

8. 2004 ASC Salary and Benefits Survey, Federated Ambulatory Surgery Association; 2004.

9. Oxford Outcomes ASC Impact Analysis; 2010.

10. Outpatient Pulse Report. Press Ganey Associates; 2008.

11. ASCA Outcomes Monitoring Project, 3rd Quarter 2011. 